\title{
Impedance spectroscopy as a probe for the degradation of organic light-emitting diodes
}

Cite as: J. Appl. Phys. 107, 054501 (2010); https://doi.org/10.1063/1.3294642

Submitted: 08 October 2009 . Accepted: 17 December 2009 . Published Online: 02 March 2010

Stefan Nowy, Wei Ren, Andreas Elschner, Wilfried Lövenich, and Wolfgang Brütting

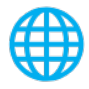

\section{ARTICLES YOU MAY BE INTERESTED IN}

Negative capacitance in organic light-emitting diodes

Applied Physics Letters 86, 073509 (2005); https://doi.org/10.1063/1.1865346

Nonradiative recombination centers and electrical aging of organic light-emitting diodes:

Direct connection between accumulation of trapped charge and luminance loss

Journal of Applied Physics 93, 1108 (2003); https://doi.org/10.1063/1.1531231

Analyzing degradation effects of organic light-emitting diodes via transient optical and electrical measurements

Journal of Applied Physics 117, 215502 (2015); https://doi.org/10.1063/1.4921829

Lock-in Amplifiers ... and more, from DC to $600 \mathrm{MHz}$

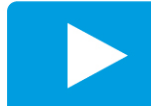

Watch 


\title{
Impedance spectroscopy as a probe for the degradation of organic light-emitting diodes
}

\author{
Stefan Nowy, ${ }^{1, a)}$ Wei Ren, ${ }^{1}$ Andreas Elschner, ${ }^{2}$ Wilfried Lövenich, ${ }^{2}$ and \\ Wolfgang Brütting ${ }^{1, b)}$ \\ ${ }^{1}$ Institute of Physics, University of Augsburg, Augsburg 86159, Germany \\ ${ }^{2}$ H. C. Starck Clevios GmbH, Chemiepark Geb. B202, Leverkusen 51368, Germany
}

(Received 8 October 2009; accepted 17 December 2009; published online 2 March 2010)

\begin{abstract}
Impedance spectroscopy is a powerful method for characterizing the electrical properties of materials and their interfaces. In this study we use capacitance measurements to investigate the degradation of electrically aged bottom-emitting organic light-emitting diodes with different polymeric hole injection layers. The devices comprise a heterojunction between a hole transporting triphenyl-diamine and an electron transporting and green emitting aluminum chelate complex $\left[\mathrm{Alq}_{3}\right.$, tris-(8-hydroxyquinoline) aluminum]. A detailed analysis of the capacitance as function of frequency and dc bias yields information about trapped and interfacial charges as well as the dynamics of injected charges. We find that the loss of luminance and the increase in drive voltage of stressed devices is accompanied by a deterioration of hole injection and the formation of positively charged quenching centers at or close to the organic heterojunction. Using a new polymeric hole injection layer leads to improved device stability. (C) 2010 American Institute of Physics. [doi:10.1063/1.3294642]
\end{abstract}

\section{INTRODUCTION}

Impedance spectroscopy (IS) is a powerful method for characterizing many of the electrical properties of materials and their interfaces, e.g., in inorganic semiconductor devices and also for studies of solid-liquid interfaces. ${ }^{1,2}$ It is employed to investigate the dynamics of bound or mobile charge in the bulk or interfacial regions, with the great advantage of being a nondestructive analysis. In literature, IS has been successfully applied to a variety of issues for organic electronics. Capacitance-voltage $(C-V)$ measurements were used to extract the acceptor dopant concentration in polymeric Schottky diodes. ${ }^{3}$ Additionally the energetic depth and the density of states created by the doping of conjugated polymers were extracted from temperature dependent investigations. ${ }^{4}$ The trap and transport states distributions for hole transport in these materials were determined by transient capacitance measurements. ${ }^{5}$ Furthermore, the dynamics of injected charge carriers was investigated under space charge limited current conditions, i.e., under forward bias, both for polymeric ${ }^{6}$ and small molecule based devices. More recently, it was shown that $C-V$ analysis in single carrier devices can also yield information about injection barriers and the built-in voltage resulting from different metal contacts. $^{8}$ Going to double carrier devices, such as organic light-emitting diodes (OLEDs) or solar cells, additional features related to the dynamics of electron-hole pairs can be monitored using IS. It was shown, e.g., that charge carrier recombination leads to the appearance of negative capacitance. ${ }^{9}$

In heterolayer devices, as discussed in this study, qualitatively new features can arise from the presence of an inter-

\footnotetext{
a)Electronic mail: stefan.nowy@physik.uni-augsburg.de.

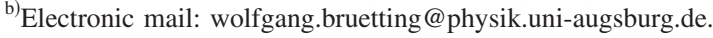

nal organic-organic interface. It was shown earlier by our group that in OLEDs comprising a heterointerface between a hole transporting triphenyl-diamine and an electron transporting aluminum chelate complex a fixed interfacial charge can be detected by applying capacitance-voltage measurements. ${ }^{10}$ In this paper, we will demonstrate how this phenomenon can be utilized to obtain information about changes at interfaces caused by device degradation under electrical operation. For this purpose, we compared devices with different polymeric hole injection layers (HILs), Clevios $^{\mathrm{TM}}$ P AI4083 (also commonly known as PEDOT/PSS) and Clevios $^{\text {TM }}$ HIL1.3. Both materials are commercially available products provided by H. C. Starck Clevios GmbH, Leverkusen. These polymers were introduced as HILs in small molecular OLEDs and led to significantly different operational stabilities. We will show how IS can be used to identify and separate different sources of degradation and discuss possible reasons.

\section{EXPERIMENTAL}

In this paper we use a well-known OLED stack comprising a hole and an electron transporting compartment, respectively (Fig. 1). The substrates for the bottom-emitting

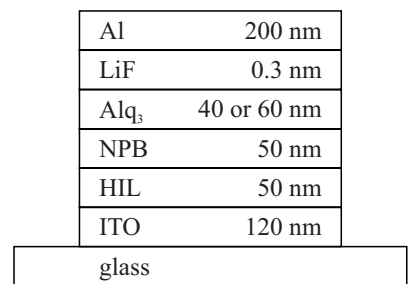

(a)

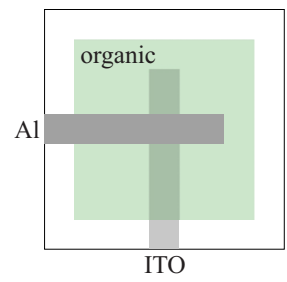

(b)
FIG. 1. (Color online) Bottom-emitting OLED stack with different HILs: AI4083 and HIL1.3 (left) and simplified crossbar layout (right). 
OLEDs consist of glass with a patterned, $120 \mathrm{~nm}$ thick indium tin oxide (ITO) layer, which is cleaned with standard procedures. On top of ITO two different HILs were used: Clevios $^{\mathrm{TM}} \mathrm{P}$ AI4083, an aqueous dispersion of poly $(3,4)$ ethylendioxythiophene (PEDOT) and poly(styrenesulfonate) (PSS) in a ratio of 1:6 by weight and Clevios ${ }^{\mathrm{TM}}$ HIL1.3 as a new PEDOT-containing dispersion for HILs provided by $\mathrm{H}$. C. Starck. ${ }^{11}$ The organic semiconductor heterojunction consists of $50 \mathrm{~nm}$ of $\mathrm{N}, \mathrm{N}^{\prime}$-di(naphtalene-1-yl)-N, $\mathrm{N}^{\prime}$ -diphenyl-benzidine (NPB) as hole transport layer (HTL) in combination with 40 or $60 \mathrm{~nm}$ of tris-(8-hydroxyquinoline) aluminum $\left(\mathrm{Alq}_{3}\right)$ as the electron transport layer (ETL). The emission zone is located at the $\mathrm{NPB} / \mathrm{Alq}_{3}$-interface. Both HILs were spincast onto the cleaned substrate and dried on a hot plate at $200{ }^{\circ} \mathrm{C}$ for $5 \mathrm{~min}$ in ambient air. The other organic materials (NPB and $\mathrm{Alq}_{3}$ ) were deposited through shadow masks in a high vacuum chamber (base pressure $<5 \times 10^{-7}$ mbar). As cathode a $0.3 \mathrm{~nm}$ thin LiF layer covered by $200 \mathrm{~nm} \mathrm{Al}$ was deposited by thermal evaporation in another chamber at $1 \times 10^{-6}$ mbar. The area of the OLED pixel defined by a crossbar layout was $4 \mathrm{~mm}^{2}$. Finally, the samples were encapsulated in a nitrogen glovebox by covering them with another glass slide sealed by epoxy resin.

All electrical measurements were performed under ambient conditions at room temperature. Current densityvoltage-luminance $(j-V-L)$ characteristics were recorded via a computer-controlled source-measure unit (Keithley 2400) and an electrometer (Keithley 6514) monitoring the photocurrent of a calibrated Si photodiode (EG\&G C30809E). The lifetime measurements were conducted in $\mathrm{N}_{2}$-environment. All devices were driven at constant current density $\left(48 \mathrm{~mA} / \mathrm{cm}^{2}\right)$. The increase in voltage and the decrease in luminance were monitored over time by a homemade setup addressing up to 24 devices simultaneously. Impedance measurements on pristine and aged devices were performed using a frequency response analyzer (Solartron SI 1260 Impedance/Gain-Phase Analyzer) combined with a dielectric interface (Solartron 1296). This setup allows frequency sweeps covering a range from $10^{-2}$ to $10^{7} \mathrm{~Hz}$. The ac oscillator level is set to $100 \mathrm{mV}_{\text {rms }}$ for all measurements and the superimposed dc bias is varied between -4 and $+6 \mathrm{~V}$. Contact angles were determined with an EasyDrop instrument from Krüss at room temperature.

\section{RESULTS AND DISCUSSION}

\section{A. Methodology}

In an IS experiment, a small, alternating electrical signal $V_{\text {ac }}(t)=V_{1} \cdot \sin (2 \pi f \cdot t)$ with small amplitude $V_{1}$ and frequency $f$ is applied to the sample in addition to a constant bias voltage $V_{\mathrm{dc}}=V_{0}$, resulting in a total applied voltage of

$$
V=V_{0}+V_{1} \cdot \sin (2 \pi f \cdot t) \text {. }
$$

$V_{0}$ serves as a handle to change the working point on the $j$ - $V$ characteristics. The response of the sample is measured as the resulting alternating current $I_{\mathrm{ac}}(t)=I_{1} \cdot \sin (2 \pi f \cdot t+\varphi)$, where $\varphi$ is a phase shift between voltage and current. The complex impedance $\hat{Z}(f)$ is defined as the ratio of the voltage and current response in complex notation $\hat{V}$ and $\hat{I}$, respectively, including their mutual phase shift:

$$
\hat{Z}(f)=\frac{\hat{V}}{\hat{I}}=\operatorname{Re}(\hat{Z})+\mathrm{i} \cdot \operatorname{Im}(\hat{Z}) .
$$

The modulus $|\hat{Z}|$ and the phase shift $\varphi$ therefore are given by

$$
|\hat{Z}|=\sqrt{\operatorname{Re}^{2}(\hat{Z})+\operatorname{Im}^{2}(\hat{Z})} \quad \text { and } \quad \varphi=\arctan \left(\frac{\operatorname{Im}(\hat{Z})}{\operatorname{Re}(\hat{Z})}\right),
$$

respectively. There are different equivalent representations of the complex impedance, however, the most intuitive one for semiconductor devices is the capacitance $C$, defined as

$$
C=\frac{1}{2 \pi f} \cdot \frac{-\operatorname{Im}(\hat{Z})}{\operatorname{Re}^{2}(\hat{Z})+\operatorname{Im}^{2}(\hat{Z})} .
$$

The complementary quantity is the dielectric loss (the conductance $G$ divided by the angular frequency $\omega$ ),

$$
\frac{G}{\omega}=\frac{1}{2 \pi f} \cdot \frac{\operatorname{Re}(\hat{Z})}{\operatorname{Re}^{2}(\hat{Z})+\operatorname{Im}^{2}(\hat{Z})},
$$

which will not be discussed further in this paper.

The data obtained by IS can be represented in several different forms, each especially suited for the topic of interest. Here we are using plots of capacitance-frequency $(C-f)$ and capacitance-voltage $(C-V)$ to extract information on charge carrier dynamics and interfacial charges, and how they are affected by OLED aging. In the following, the basic ideas behind these data representations are explained.

The OLEDs under investigation consist of two undoped organic semiconductor materials, NPB and $\mathrm{Alq}_{3}$. Each of these two layers has a (low, but nonvanishing) conductivity and a dielectric constant and can thus be represented by a resistor $R$ and a capacitor $C$ connected as a parallel $R C$ element. We obtain the complex impedance $\hat{Z}$ for one layer as

$$
\hat{Z}_{R C}=\frac{1}{\frac{1}{R}+\mathrm{i} \omega C},
$$

therein $\omega=2 \pi f$ denotes the angular frequency.

To model the OLED stack we consider two of these $R C$ elements connected in series, representing the holeconducting NPB layer (HTL) and the electron-conducting $\mathrm{Alq}_{3}$ layer (ETL), having the impedance

$$
\hat{Z}=\hat{Z}_{R_{1} C_{1}}+\hat{Z}_{R_{2} C_{2}} \equiv \hat{Z}_{\mathrm{HTL}}+\hat{Z}_{\mathrm{ETL}} .
$$

If an alternating voltage according to Eq. (1) is applied with varying frequency $f$ we find two plateaus in the capacitance for low and high frequency and a transition region around a characteristic relaxation frequency $f_{\mathrm{r}}$. According to Eqs. (4) and (7) the limiting case for the total capacitance $C_{\text {tot }}$ in the high frequency range is 


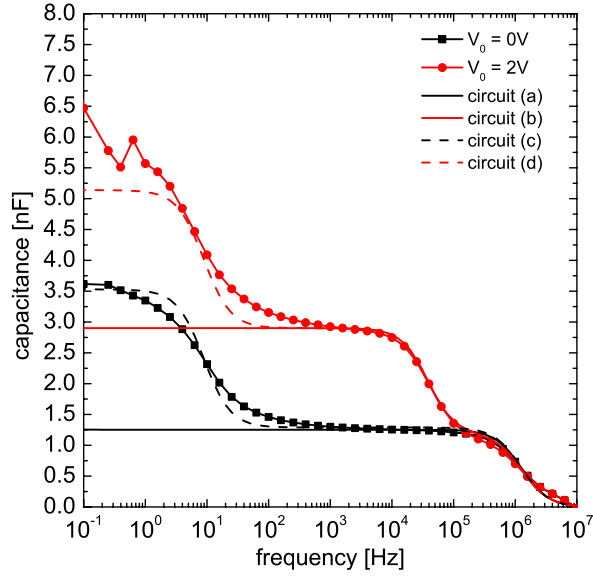

FIG. 2. (Color online) Capacitance-frequency plot for the OLED of Fig. 1 with HIL1.3 and $40 \mathrm{~nm} \mathrm{Alq}_{3}$ at zero (0 V, black line with black squares) and forward bias ( $2 \mathrm{~V}$, red line with red circles). Additionally, simulations of different equivalent circuits (see Fig. 3) are shown.

$$
\lim _{f \rightarrow \infty} C_{\text {tot }}=\frac{C_{1} C_{2}}{C_{1}+C_{2}},
$$

which is just the total (geometrical) capacitance of two capacitors in series. However, the low frequency limit (which corresponds to the dc regime) also depends on the values of the two resistors:

$$
\lim _{f \rightarrow 0} C_{\text {tot }}=\frac{R_{1}^{2} C_{1}+R_{2}^{2} C_{2}}{\left(R_{1}+R_{2}\right)^{2}} .
$$

The transition between these two limiting cases happens at the relaxation frequency $f_{\mathrm{r}}$ which is given by ${ }^{12}$

$$
f_{\mathrm{r}}=\frac{1}{2 \pi \tau_{\mathrm{r}}}=\frac{1}{2 \pi} \cdot \frac{R_{1}+R_{2}}{R_{1} R_{2} \cdot\left(C_{1}+C_{2}\right)} .
$$

If we now assume that one of the two semiconducting layers becomes significantly less resistive, e.g., $R_{1} \ll R_{2}$, Eqs. (9) and (10) yield

$$
\lim _{f \rightarrow 0} C_{\mathrm{tot}}=C_{2} \quad \text { and } \quad f_{\mathrm{r}}=\frac{1}{2 \pi} \cdot \frac{1}{R_{1} \cdot\left(C_{1}+C_{2}\right)},
$$

respectively.

Figure 2 shows experimental $C$ - $f$ curves for an OLED with the structure $\operatorname{ITO}(120 \mathrm{~nm}) / \mathrm{HIL}(50 \mathrm{~nm}) /$ $\operatorname{NPB}(50 \mathrm{~nm}) / \mathrm{Alq}_{3}(40 \mathrm{~nm}) / \operatorname{LiF}(0.3 \mathrm{~nm}) / \operatorname{Al}(200 \mathrm{~nm})$ together with simulated $C$ - $f$ characteristics for four different equivalent circuits (a)-(d) (see Fig. 3). Additionally, to account for the nonvanishing ITO lead resistance an additional series resistance of the order of $100 \Omega$ is included, leading to a roll off from the geometrical capacitance to zero at frequencies of about $1 \mathrm{MHz}$. Circuits (a) and (b) show two different situations. In (a) both the HTL and the ETL are reverse biased and have very large resistance. Thus one obtains just the geometric capacitance of both layers in series. This situation corresponds to the measured $C$ - $f$ characteristics at zero or negative bias. However, if one layer becomes significantly less resistive than the other one [circuit (b)], e.g., by carrier injection into the HTL, a transition from the geometrical capacitance to the capacitance of the remaining (a)

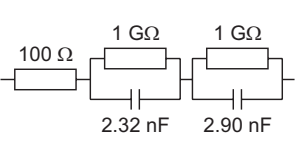

(c)

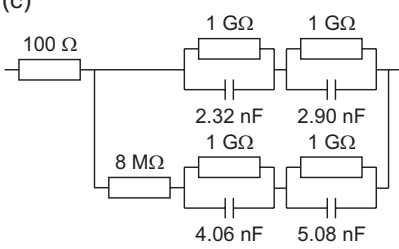

(b)

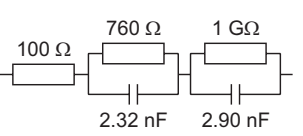

(d)

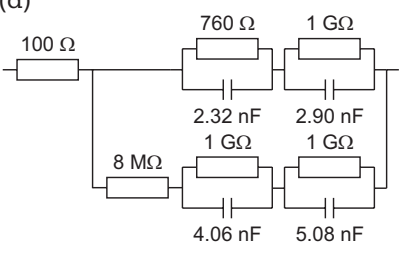

FIG. 3. Equivalent circuits for the simulations shown in Fig. 2. Circuit (a): reverse bias condition. Circuit (b): hole injection regime. Circuit (c): reverse bias condition with additional leakage current path. Circuit (d): hole injection regime with additional leakage current path. The values of the capacitance for the HTL and ETL layer were calculated from the active pixel area and the respective layer thicknesses using a relative dielectric constant of 3.28. For estimating the values for the leakage pathway the capacitance of both layers was scaled up with the same factor 1.75 corresponding to an effectively increased device area. The resistance of $8 \mathrm{M} \Omega$ represents the lateral (in-plane) conduction through the HIL.

highly resistive ETL occurs at the relaxation frequency given by Eq. (11). For the chosen parameter values, $f_{\mathrm{r}}$ is in the range of $10^{4}$ to $10^{5} \mathrm{~Hz}$.

Figure 2 includes another set of $C$ - $f$ curves for circuits (c) and (d) where an additional parasitic double $R C$ element is assumed in parallel to the active OLED (see Fig. 3). These circuits show in addition to the already described relaxation step a second step in the capacitance at about $10 \mathrm{~Hz}$. As discussed in detail in another publication, ${ }^{13}$ this feature is related to a leakage current pathway originating from the fact that in a crossbar architecture without structured organic semiconductor there is an additional parasitic diode in parallel to the active OLED pixel. Comparison to experimental data shows that this model yields an excellent description of measured $C$ - $f$ curves, thus containing the essential circuit elements.

From this comparison between experimental and simulated $C$ - $f$ curves one has to conclude that between 0 and $2 \mathrm{~V}$ applied bias, i.e., well below the turn-on voltage of the OLED, a redistribution of the electric field inside the device occurs, which is accompanied by the relaxation step in the $10-100 \mathrm{kHz}$ range. Beyond the good description with equivalent circuits, however, there remains the question by which physical processes this feature is caused. To clarify this issue, one has to look at capacitance-voltage measurements as displayed in Fig. 4 for a fixed frequency of $100 \mathrm{~Hz}$. At negative bias we see a constant capacitance, corresponding to the geometric capacitance of the device.

$$
C_{\mathrm{geo}}=\frac{\epsilon_{0} \epsilon_{\mathrm{r}} A}{d}
$$

where $\epsilon_{0}$ is the permittivity of free space, $\epsilon_{\mathrm{r}} \approx 3.28$ [calculated from the $C-f$ measurements and the corresponding layer thicknesses, valid for both HTL and ETL (Ref. 14)] is the relative dielectric constant, $A$ is the area of the OLED pixel, and $d=d_{\mathrm{NPB}}+d_{\mathrm{Alq}_{3}}$ is the combined thickness of the HTL and ETL. However, if the bias becomes larger than a 


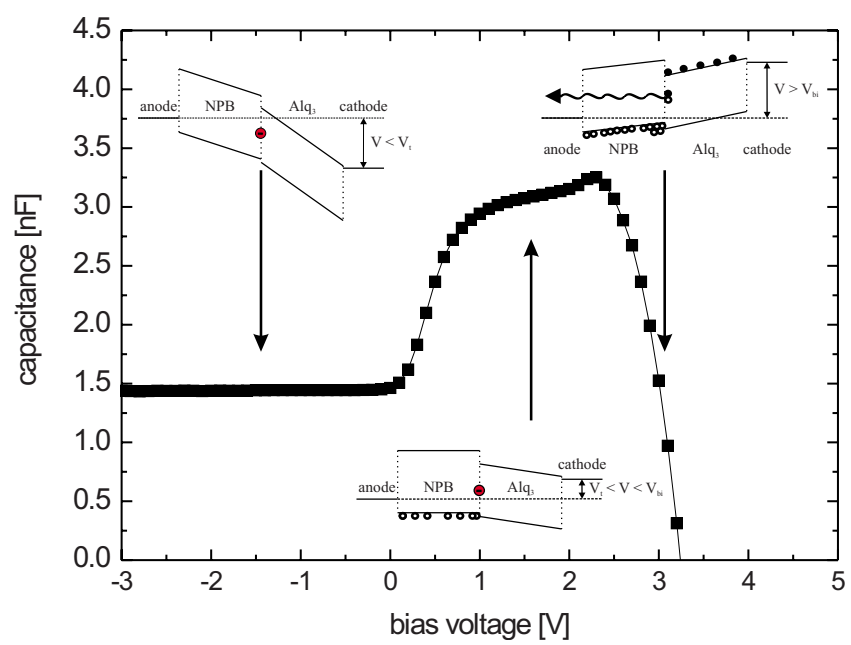

FIG. 4. (Color online) Capacitance-voltage plot for the OLED with HIL1.3 and $40 \mathrm{~nm} \mathrm{Alq}{ }_{3}$ at an applied frequency of $f=100 \mathrm{~Hz}$. Additionally, simplified band diagrams are shown, from left to right: reverse bias condition $\left(V<V_{\mathrm{t}}\right)$, hole injection regime $\left(V_{\mathrm{t}}<V<V_{\mathrm{bi}}\right)$, hole and electron injection regime, and recombination $\left(V>V_{\mathrm{bi}}\right)$.

transition voltage $V_{\mathrm{t}}$ (here $\approx 0.1 \mathrm{~V}$ ) holes are injected to the device, raising the capacitance, as they accumulate at the $\mathrm{NPB} / \mathrm{Alq}_{3}$-interface and the capacitance increases toward the value of the $\mathrm{Alq}_{3}$ layer:

$$
C_{\mathrm{Alq}_{3}}=\frac{\epsilon_{0} \epsilon_{\mathrm{r}} A}{d_{\mathrm{Alq}_{3}}} .
$$

At the built-in voltage $V_{\text {bi }}$, indicated by the peak in $C$ at 2.3 $\mathrm{V}$, electron injection from the cathode side into the device starts. ${ }^{32}$ As electrons and holes recombine to generate light, charge is annihilated, thus the differential capacitance decreases rapidly with increasing potential and even becomes negative. This is also evident from $C-f$ characteristics at low enough frequencies and bias voltages above $V_{\mathrm{bi}}$ (not shown here). We note that it has already been shown by Ehrenfreund et $a l^{9}{ }^{9}$ that the negative contribution to the capacitance occurs only for bipolar injection. Thus its appearance in our devices can be taken as an indication for the onset of electron injection.

The increase in the capacitance toward $C_{\mathrm{Alq}_{3}}$ at a voltage $V_{\mathrm{t}}$ lower than the built-in voltage requires that a negative interfacial charge $Q_{\text {if }}$ is present at the HTL/ETL-interface. ${ }^{10,14}$ Its value can be calculated from the following equation: ${ }^{14}$

$$
\sigma_{\mathrm{if}}=Q_{\mathrm{if}} / A=\frac{\epsilon_{0} \epsilon_{\mathrm{r}}}{d_{\mathrm{Alq}_{3}}} \cdot\left(V_{\mathrm{t}}-V_{\mathrm{bi}}\right) .
$$

With $\epsilon_{\mathrm{r}} \approx 3.28, A=4 \mathrm{~mm}^{2}$, and $d_{\mathrm{Alq}_{3}}=40 \mathrm{~nm}$ we obtain $Q_{\mathrm{if}}$ $\approx-6.3 \mathrm{nC}$ and $\sigma_{\mathrm{if}} \approx-1.58 \mathrm{mC} / \mathrm{m}^{2}$, corresponding to 9.9 $\times 10^{11}$ charges $/ \mathrm{cm}^{2}$. Similar values, also for other material combinations and obtained with other measurement techniques, have been published. ${ }^{10,15-17}$ Recent studies show that the origin of this negative charge at the interface is due to dipole moment ordering in the $\mathrm{Alq}_{3}$ layer during film preparation. ${ }^{17}$ The polar $\mathrm{Alq}_{3}$ molecules grow with a preferential orientation on the NPB layer, so that there is a net negative charge at the HTL/ETL interface. Taking the dipole moment of 4.1 D for $\mathrm{Alq}_{3}$, one can estimate the degree of orientation of the dipole moments to be about $1 \%$. Given the fact that many molecular materials used in organic electronics are polar, we expect that similar interface charges could exist in other heterolayer devices, too.

With this microscopic explanation for the redistribution of the electric field as function of the applied bias in mind, one can now also interpret the observed relaxation frequency, where the capacitance changes from $C_{\mathrm{Alq}_{3}}$ to the lower geometrical value. As explained above, the observation of an enhanced capacitance for a heterolayer device requires that the HTL is significantly less resistive than the ETL. This means that holes must be injected into NPB and be transported to the interface to $\mathrm{Alq}_{3}$ well before electron injection starts. However, this process is not arbitrarily fast but limited by the occurrence of an injection barrier and/or the mobility in the NPB layer. Thus the relaxation frequency $f_{\mathrm{r}}$ can be taken as a measure of how good hole injection into the HTL and/or hole transport to the HTL/ETL interface is.

In the following we will use the bias and frequency dependent redistribution of the internal electric field inside the heterolayer OLEDs, being indicative of fixed negative interfacial charges and mobile injected positive charges, to monitor changes in hole injection and the creation of additional charges upon OLED degradation.

\section{B. Comparison of different hole injection layers}

Before discussing stability issues of both types of OLEDs with different HIL, we will compare current densityvoltage $(j-V)$ characteristics and impedance spectra of pristine samples. Figure 5 shows $j-V$ characteristics of OLEDs with $40 \mathrm{~nm} \mathrm{Alq}$ and the two different HILs. Apart from some differences in the leakage current between 0 and $2 \mathrm{~V}$, which can vary from sample to sample, both OLEDs show virtually identical behavior in forward direction. In particular, the current starts to rise exponentially at about $2.3 \mathrm{~V}$ and at the same voltage light emission sets in. Also shown in Fig. 5 is the efficiency which for both devices reaches values of about $2.75 \mathrm{~cd} / \mathrm{A}$ comparable to published data for this device structure. $^{18}$

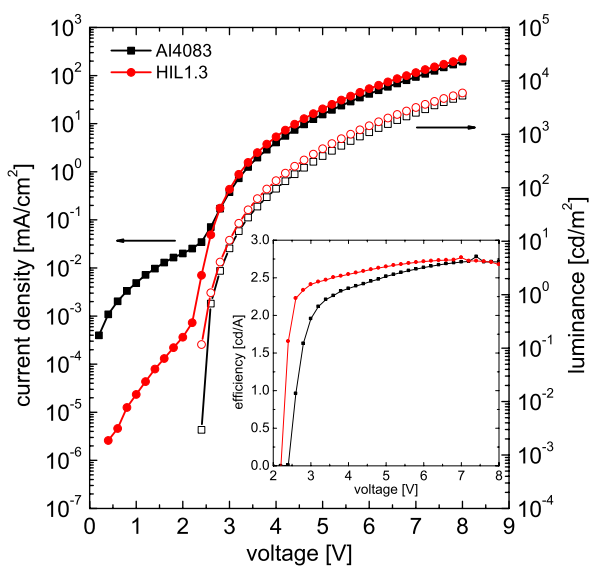

FIG. 5. (Color online) $j-V-L$ characteristics and efficiency of pristine OLEDs with different HIL and $40 \mathrm{~nm} \mathrm{Alq} \mathrm{q}_{3}$. 

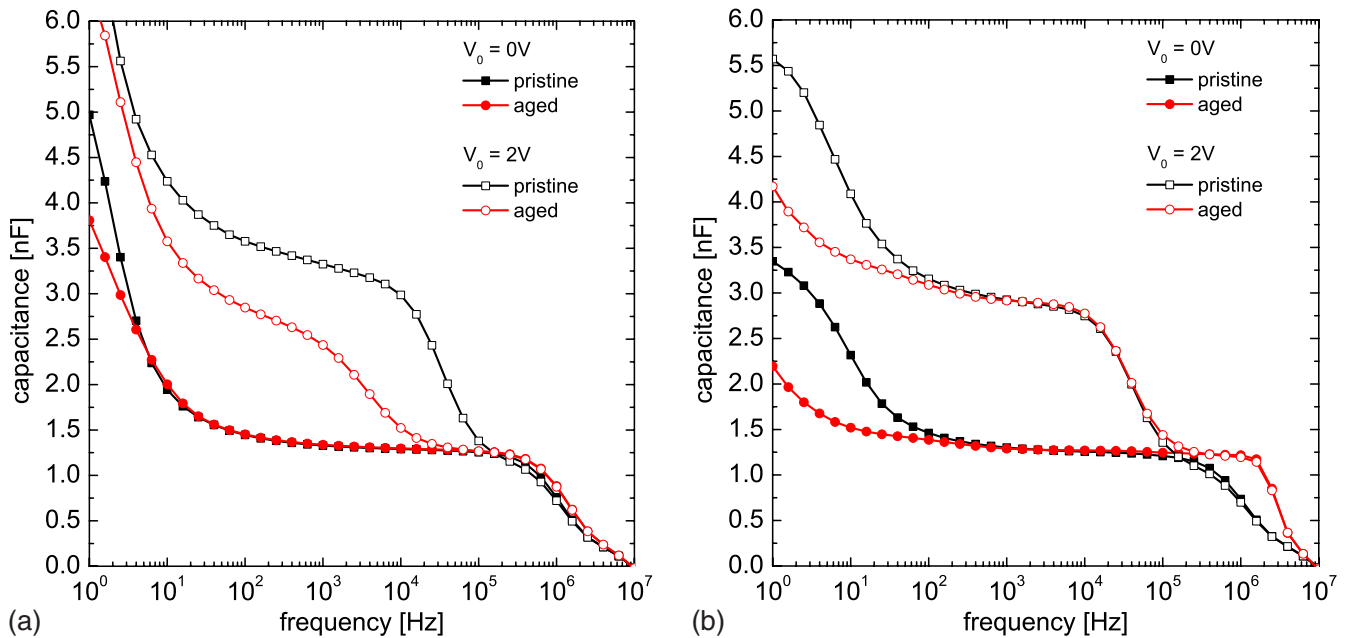

FIG. 6. (Color online) Capacitance-frequency plot for pristine (black symbols and lines) and aged (red symbols and lines) samples with (a) AI4083 and (b) HIL1.3 as HIL and different applied bias $V_{0}=0$ and $2 \mathrm{~V}$. Aging was performed under constant current drive at $j=48 \mathrm{~mA} / \mathrm{cm}^{2}$ for $215 \mathrm{~h}(\mathrm{AI} 4083)$ and $250 \mathrm{~h}$ (HIL1.3), respectively. (Alq ${ }_{3}$ thickness: $40 \mathrm{~nm}$.)

Figure 6 shows the frequency dependent capacitance at different dc bias values for pristine samples. Qualitatively, both types of devices show the behavior discussed in the previous section (Fig. 2). For negative bias (including $V_{0}$ $=0 \mathrm{~V}$ ) both organic layers are highly resistive and one measures just the geometrical capacitance. (There is some difference in the low-frequency increase between devices with different HILs, indicating different parasitic stray capacitance resp. leakage current pathway, but this is not of further interest here.) However, as soon as the bias voltage exceeds the transition voltage $V_{\mathrm{t}}(\approx 0.5 \mathrm{~V}$ for AI4083 as HIL and $\approx 0.1 \mathrm{~V}$ for HIL1.3, see below), the measured capacitance corresponds to the value of the $\mathrm{Alq}_{3}$ layer. Again, there is some difference between both devices, the one with AI4083 having slightly higher values of $C$, which could be due to a slightly thinner $\mathrm{Alq}_{3}$ layer. More remarkable, however, is the fact that the relaxation frequency $f_{\mathrm{r}}$ for a bias of $2 \mathrm{~V}$ is identical for both devices, indicating that the injection of holes into the HTL and/or their transport to the HTL/ETL interface is the same for both HILs. This is surprising as the work function of the two materials determined by ultraviolet photoelectron spectroscopy was found to be significantly different, 5.1 and $5.7 \mathrm{eV}$ for AI4083 and HIL1.3, respectively. ${ }^{19}$ However, earlier work indicates that the energy barrier for hole injection is not solely determined by the difference in work function of the HIL and the HOMO level of the HTL. The formation of a dipole layer at the interface HIL/HTL shifts the vacuum levels significantly in its relative positions especially for PEDOT containing HILs so that the Fermi level of the latter is effectively pinned at the hole transport level (positive polaron level) in the organic material. ${ }^{20}$

Also included in Fig. 6 are $C-f$ measurements for two OLEDs with the identical layer structure as the pristine ones after operating the devices under a constant current density of $48 \mathrm{~mA} / \mathrm{cm}^{2}$ for 215 and $250 \mathrm{~h}$, respectively. While in the case of HIL1.3 there is only very little change in the overall behavior, the curve for AI4083 as HIL changes quite significantly. Its relaxation frequency at $V_{\text {bias }}=2 \mathrm{~V}$ is almost tenfold smaller than for the pristine sample which points toward a deterioration of hole injection and transport to the HTL/ ETL interface. By contrast, the relaxation frequency for the sample with HIL1.3 is unchanged. The only changes observable in this device are less effects of stray capacitance at low frequency and a slightly smaller series resistance, leading to a roll off beyond $1 \mathrm{MHz}$.

Complementary to $C$ - $f$ one can measure the capacitancevoltage dependence at a fixed frequency. This is shown for $f=100 \mathrm{~Hz}$ in Fig. 7. Both samples in the pristine state show similar values of the transition voltage $V_{\mathrm{t}}$ where hole injection into the HTL starts. As $V_{\mathrm{t}}-V_{\mathrm{bi}}$ is proportional to the thickness of the $\mathrm{Alq}_{3}$ layer [Eq. (14)], the slightly more positive value of $V_{\mathrm{t}}$ for the sample with AI4083 as HIL (meaning that $V_{\mathrm{t}}-V_{\mathrm{bi}}$ is smaller) could indicate that unintentionally the $\mathrm{Alq}_{3}$ layer was grown thinner as compared to the sample with HIL1.3. This would also be in agreement with a larger value of $C_{\mathrm{Alq}_{3}}$ observed in $C$ - $f$. For bias voltages larger than $V_{\mathrm{t}}$ in both samples the HTL is accumulated with holes so that one measures only the ETL capacitance. Remarkably, at the same voltage $V_{\text {peak }} \approx 2.3 \mathrm{~V}$, where the $j$-V characteristics start to increase exponentially, there is a small peak in the $C-V$ curves for both pristine samples, indicating that the built-in voltage is identical.

Figure 7 also compares $C$ - $V$ characteristics of pristine and aged devices (same as in Fig. 6). Again the sample with HIL1.3 does not exhibit strong changes, whereas the one with AI4083 as HIL shows a pronounced shift of both $V_{\mathrm{t}}$ and $V_{\text {peak }}$ toward more positive values, indicating that the negative interfacial charge at the HTL/ETL interface has decreased and that electron injection now requires higher voltages.

For a more detailed analysis of device degradation and the concomitant changes in impedance spectra, we performed long-term aging of another series of devices with both HILs. In these devices an $\mathrm{Alq}_{3}$ thickness of $60 \mathrm{~nm}$ was used. Figure 8 shows drive voltage and luminance versus aging time for four samples of each type operated at a constant current density of $48 \mathrm{~mA} / \mathrm{cm}^{2}$ over different periods of time. As the measured pixels were partially located on dif- 

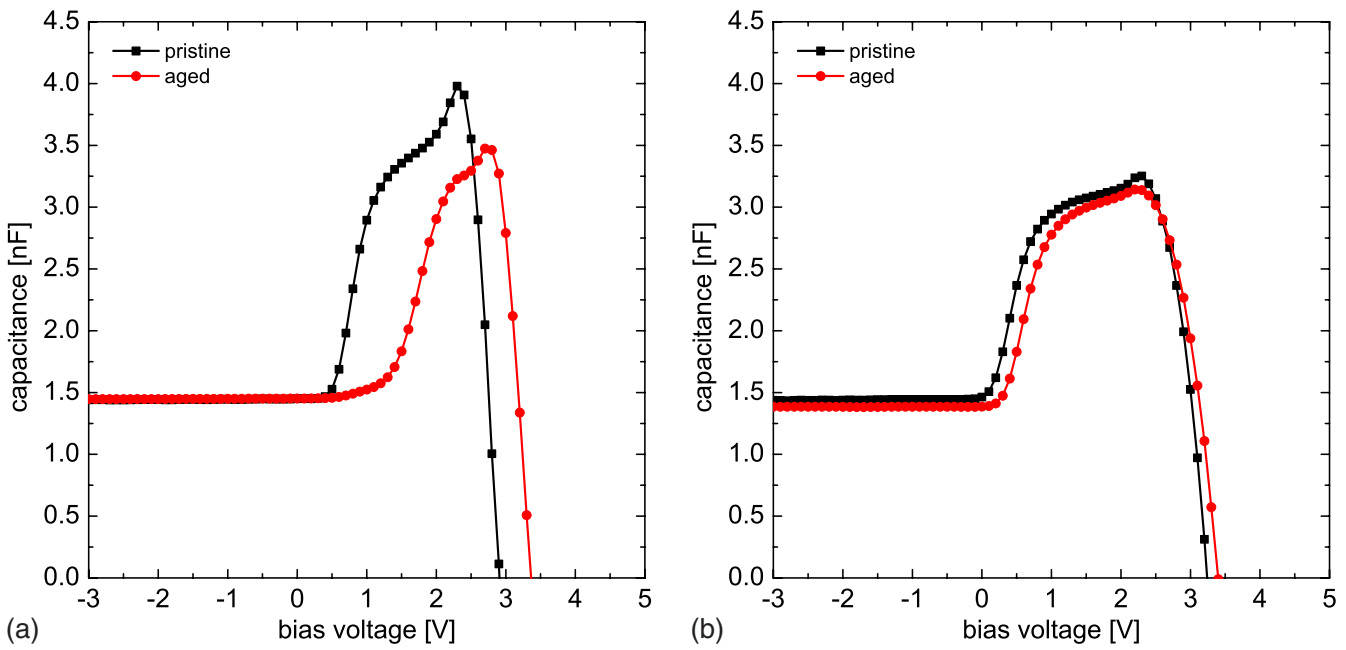

FIG. 7. (Color online) $C$ - $V$ characteristics of pristine and aged OLEDs with (a) AI4083 and (b) HIL1.3 as HIL. Aging was performed under constant current drive at $j=48 \mathrm{~mA} / \mathrm{cm}^{2}$ for $215 \mathrm{~h}$ (AI4083) and $250 \mathrm{~h}$ (HIL1.3), respectively. (Applied frequency: $f=100 \mathrm{~Hz}$. Alq $\mathrm{q}_{3}$ thickness: $40 \mathrm{~nm}$.)

ferent chips fabricated in different runs, there is some scattering between them, but one can nevertheless see a clear tendency. The devices with AI4083 as HIL degrade very rapidly: the luminance drops to half of its initial value after $20-40 \mathrm{~h}$ and after $200 \mathrm{~h}$ it is less than $20 \%$ for all samples. At the same time the drive voltage increases by about $2.5 \mathrm{~V}$ already after 200 h. For samples with HIL1.3, however, the loss in luminance and the increase in drive voltage are much slower: after $200 \mathrm{~h}$ the luminance is still above $70 \%$ and the extrapolated half lifetime would be larger than $500 \mathrm{~h}$ for most devices. This is a clear indication that the rapid device degradation in the former type of devices is related to the usage of AI4083 as HIL.

It is instructive to compare the capacitance-voltage characteristics of these devices as function of operation time as shown in Fig. 9 for a fixed frequency of $100 \mathrm{~Hz}$. Please note that due to the increased thickness of the $\mathrm{Alq}_{3}$ layer the curves for the pristine devices differ slightly from the ones shown in Fig. 7. As expected from Eq. (14) the transition voltage is more negative $\left(V_{\mathrm{t}} \approx-0.9 \mathrm{~V}\right)$ and the $\mathrm{Alq}_{3}$ capacitance is smaller. Otherwise the behavior is as discussed above for pristine samples and, in particular, identical for both types of HILs. For the aged samples, however, there is a remarkable difference: OLEDs with AI4083 have a rapid shift of the transition voltage toward more positive values and for aging times longer than $150 \mathrm{~h} V_{\mathrm{t}}$ even exceeds the built-in voltage of the pristine sample. At the same time the relaxation frequency decreases very rapidly (not shown here). By contrast, devices with HIL1.3 display a much slower shift of the transition voltage and even after $450 \mathrm{~h}$ of constant driving $V_{\mathrm{t}}$ is still well below the built-in voltage.

\section{Discussion}

It is generally known that the luminance of OLEDs is decreasing with operating time, temperature, and current load, hence their lifetime unfortunately is still limited. There have been various reports about possible degradation mechanisms, e.g., migration of mobile ions in the device, ${ }^{21}$ reorientation of molecular dipoles, ${ }^{22,23}$ chemical degradation due to oxygen ${ }^{24}$ and/or water, ${ }^{25}$ or instability of charge-carrying species (e.g., $\left.\mathrm{Alq}_{3}\right) .{ }^{26}$ However, as these reports use different
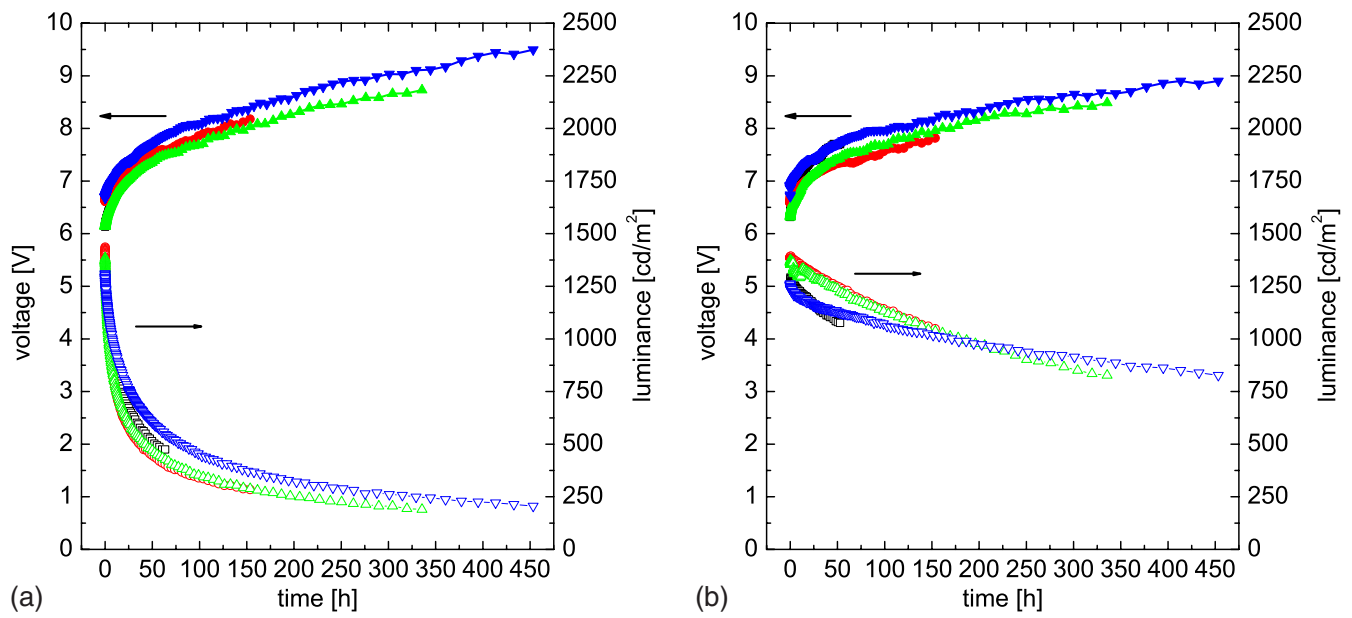

FIG. 8. (Color online) Voltage increase and luminance loss of OLEDs with (a) AI4083 and (b) HIL1.3 as HIL for constant current driving at $j$ $=48 \mathrm{~mA} / \mathrm{cm}^{2}$. (Closed symbols: voltage; open symbols: luminance; $\mathrm{Alq}_{3}$ thickness: $60 \mathrm{~nm}$.) 

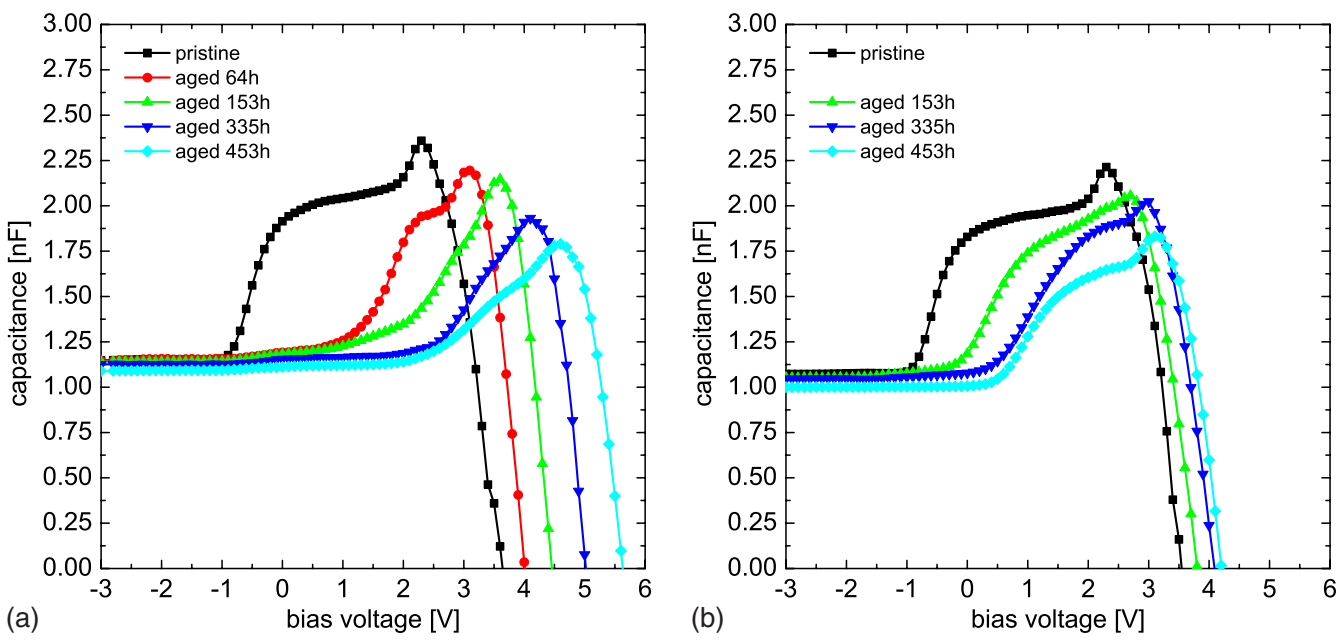

FIG. 9. (Color online) Capacitance-voltage plots for the different aging times. (a) AI4083 and (b) HIL1.3 as HIL. (Applied frequency: $f=100 \mathrm{~Hz}$; Alq 3 thickness: $60 \mathrm{~nm}$.)

materials and material combinations, it might be that one or the other mechanisms is more pronounced in the OLED stack of interest.

It is furthermore important to note that so far only very few experimental techniques have been employed to study OLED degradation. Among them the most direct method is the measurement of the temporal development of the voltage and the light output under electrical driving (either continuous constant current or alternating drive conditions with constant forward current for one half-cycle and constant reverse bias for the other half). This directly yields the loss of luminance and thus efficiency together with the increase in the voltage required to drive the current, which is a measure of how the current-voltage characteristics changes by electrical driving. In order to separate effects on charge transport (e.g., trapping) from effects affecting radiative recombination, photoluminescence has been studied on electrically aged devices. $^{27}$ Recently, chemical analysis of the degradation products of aged OLEDs by breaking the encapsulation and extracting the organic compounds in solvents suitable for chromatographic analysis techniques has been performed. ${ }^{28}$

Another class of nondestructive techniques, which is well known from inorganic semiconductor devices, makes use of the capacitive response of OLEDs under reverse bias conditions. In so-called voltammetric $j-V$ measurements the applied voltage is ramped with a high scan rate of typically $50 \mathrm{~V} / \mathrm{s}$ and the displacement current $(C \cdot d V / d t)$ due to charging or discharging of the sample capacitor is measured. Therefore this technique is also known as displacement current method (DCM). ${ }^{29}$ Kondakov et al. ${ }^{16}$ were the first to apply voltammetry to study OLED degradation. Their studies confirmed the existence of negative interfacial charges in pristine $\mathrm{NPB} / \mathrm{Alq}_{3}$ heterolayer OLEDs as introduced before by us. ${ }^{10}$ Moreover, they found that upon electrical aging of these devices the interfacial charge, evident through a step in the voltammetry signal in analogy to $V_{\mathrm{t}}$ in our $C$ - $V$ measurements, is steadily decreasing and finally gets positive for longer operating times. From the observed linear relationship between the loss of luminance efficiency and the shift of the transition voltage (reflecting the sign and the amount of in- terfacial charge) they concluded that electrical aging of these OLEDs creates positive fixed charges located primarily near the HTL/ETL interface. ${ }^{16}$ They further suggested that these charged states act as nonradiative recombination centers. As far as the microscopic origin of these centers is concerned, a natural explanation was given in a series of papers by Aziz and Popovic. ${ }^{18,26,27}$ They could show that degradation of $\mathrm{NPB} / \mathrm{Alq}_{3}$ heterolayer OLEDs is mainly caused by instability of cationic $\mathrm{Alq}_{3}^{+\cdot}$, being formed by excess holes injected into the ETL. On the other hand, a recent study by Kondakov indicates that chemical degradation of the hole transporting arylamine moiety can also have a considerable contribution to OLED degradation. ${ }^{30}$ The degradation products are considered as irreversible hole traps leading to an accumulation of positive fixed charge at the HTL/ETL interface. Thus both scenarios lead to identical behavior in DCM measurements and are not distinguishable by this technique alone.

IS used in our study detects differential changes of the amount of charge in the sample capacitor as a function of frequency and bias. In principle, our $C-V$ measurements yield the same information as the voltammetric measurements discussed above. However, there is the advantage that in IS these voltage scans can be performed at (almost) any frequency accessible by the analyzer without losing signal. Furthermore, one can do $C$ - $f$ sweeps at different working points selected by the superimposed dc bias. This allows for probing the dynamics of injected carriers. The results presented here show that both types of samples in the pristine state exhibit virtually identical response. The observation of a transition from the geometrical capacitance to the capacitance of the $\mathrm{Alq}_{3}$ layer alone at a voltage $V_{\mathrm{t}}$ can only be explained by a fixed negative interfacial charge of about $1.6 \mathrm{mC} / \mathrm{m}^{2}$. As already mentioned, the origin of this charge is partial dipole moment ordering (orientational polarization) of the $\mathrm{Alq}_{3}$ molecules. ${ }^{17}$ It is thus an intrinsic property of a material consisting of polar molecules and not related to the existence of deep traps as sometimes stated in the literature. $^{31}$ As the devices are electrically aged, the transition voltage is shifting toward more positive values and there is a direct correlation between $V_{\mathrm{t}}$ and the loss of luminance 


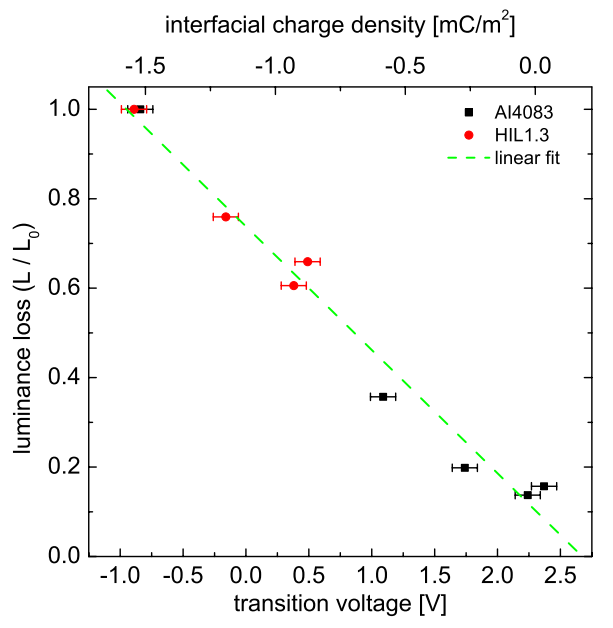

FIG. 10. (Color online) Luminance efficiency loss as a function of transition voltage (lower $x$-axis) and interfacial charge density (upper $x$-axis). Black squares (AI4083) and red circles (HIL1.3) are experimental data extracted from different OLED pixels. The green dashed line is a linear fit of all data points.

efficiency (Fig. 10) as observed also in voltammetric measurements. ${ }^{16}$ The linear fit in Fig. 10 represents all experimental data for both types of devices extremely well, suggesting that the underlying degradation mechanism is the same for both device types, but happening on different timescales. Apart from changes in the interfacial charge, IS also yields information about the dynamics of injected carriers via the relaxation frequency. Its shift toward lower frequencies with increasing aging time shows that the resistance of the HTL in the equivalent circuit must be increasing. A priori, this could be caused by either an increase in the injection barrier or a reduction in the hole mobility, e.g., by traps in the bulk of the HTL. It is likely that both effects may contribute, but obviously the HIL/HTL interface plays a decisive role in this process.

As discussed above, it is well established that the origin of the shift in transition voltage is due to the formation of positively charged defect states close to the HTL/ETL interface, nevertheless, there is the question why the devices with different HIL behave differently. Following the unstable cationic $\mathrm{Alq}_{3}$ model suggested by Aziz and Popovic ${ }^{26}$ one could speculate that in devices with HIL1.3 hole injection and transport to the ETL is impeded. However, this is not very likely already from the $j-V$ characteristics and the efficiency (Fig. 5), being the same for pristine devices. Direct evidence comes from the $C-f$ measurements shown in Fig. 6 where identical relaxation frequencies, i.e., identical resistance for the transport of holes to the HTL/ETL interface are observed. Moreover, upon degradation the relaxation frequency for the device with HIL1.3 decreases much slower as compared to AI4083 as HIL. From that point of view, one has to conclude that devices with HIL1.3 have better hole injection and transport over the entire range of device life, so the amount of holes flowing through the device is obviously not the crucial point. Since both devices in the pristine state show identical hole injection properties and identical HTL/ETL interface conditions, one has to consider another possibility having its origin in the HILs themselves.
The different rates of degradation can be explained if one assumes that the two HILs induce different speeds for the formation of positively charged nonradiative recombination centers. Probably not by directly emitting some charged species (polymeric ions are contained in both HILs), but by delivering some kind of degradation catalyst at a different rate or amount. As both HILs are spin coated from water containing dispersions a possible candidate could be water which is known to cause instability of $\mathrm{Alq}_{3} \cdot{ }^{25}$ However, since both HILs are dried on a hot plate they should not contain large quantities of water in their bulk. Additionally, in experiments with various drying conditions, no significant differences between devices with different HILs were found. Another parameter might be the acidity of the HIL being relevant for interface dipole formation and charge injection to the HTL. It does however not explain the difference between AI4083 and HIL1.3 as the acidity is very similar $(\mathrm{pH}=1.6-1.8)$ for both materials. In contrast with that, the properties of the layer surface were found to be significantly different for both HILs. Results from contact angle measurements show that HIL1.3 is less hydrophilic (contact angle with toluene $48^{\circ}$ ) as compared to AI4083, where the contact angle is almost zero. Although it needs to be verified that more hydrophobic HILs will lead to more stable HIL/HTL interfaces, in general it is reasonable to explain the observed phenomenon of reduced voltage increase and luminance drop in devices with HIL1.3 by this mechanism.

Furthermore, IS also gives a hint toward changes in electron injection by a shift in the peak voltage in the $C$ - $V$ curves (see Fig. 9). Devices with AI4083 show a shift of both $V_{\mathrm{t}}$ and $V_{\text {peak }}$ already in the early stage of degradation, whereas those with HIL1.3 have $V_{\text {peak }}$ remaining unaffected at the beginning. Preliminary data on heterolayer devices with an inorganic HIL (e.g., $\mathrm{WO}_{3}$ ) confirm this notion: ${ }^{13}$ the use of different HILs also affects the electron injection under electrical aging of the devices. There is currently very little quantitative work, ${ }^{9}$ but IS could also prove to be a very useful technique to study recombination dynamics in OLEDs and how this is affected by device degradation in the future.

\section{SUMMARY}

In this paper we used IS to study degradation in OLEDs with different polymeric HILs. We have shown that capacitance-frequency analysis is a sensitive tool to monitor charge injection and yields information especially in voltage regimes $\left(V<V_{\mathrm{bi}}\right)$, where current density-voltage measurements are not sensitive. Capacitance-voltage analysis identifies trapped or interfacial charges. In the OLED stack investigated here, a negative interfacial charge due to bulk polarization is present already in pristine devices. However, with operational lifetime of the OLED accumulation of positive charge at or near the HTL/ETL interface is effectively reducing its amount. This accumulation of positive charge at the $\mathrm{NPB} / \mathrm{Alq}_{3}$-interface directly correlates with loss in luminance, suggesting that the positive charges act as nonradiative recombination centers. Comparison of both HILs used here shows that the speed of degradation depends on the stability of the interface between the HILs and the hole trans- 
porting layer, which could be due to different surface properties of both HILs. Both types of IS measurements presented in this paper are very useful tools in the analysis of degradation processes in OLEDs and offer the possibility to contribute to the ongoing discussions about intrinsic device degradation mechanisms.

\section{ACKNOWLEDGMENTS}

The authors would like to thank the German Federal Ministry of Education and Research (BMBF) for funding part of this work under Contract Nos. FKZ 13N10474 (TOPAS) and FKZ 13N10615 (NEMO). We also thank Y. Noguchi (Chiba University, Japan) for fruitful discussions.

${ }^{1}$ Impedance Spectroscopy, edited by J. R. Macdonald (Wiley, New York, 1987).

${ }^{2}$ J. A. Garrido, S. Nowy, A. Härtl, and M. Stutzmann, Langmuir 24, 3897 (2008).

${ }^{3}$ M. Meier, S. Karg, and W. Riess, J. Appl. Phys. 82, 1961 (1997).

${ }^{4}$ J. Scherbel, P. H. Nguyen, G. Paasch, W. Brütting, and M. Schwoerer, J. Appl. Phys. 83, 5045 (1998).

${ }^{5}$ A. J. Campbell, D. D. C. Bradley, E. Werner, and W. Brütting, Synth. Met. 111-112, 273 (2000).

${ }^{6}$ H. C. F. Martens, H. B. Brom, and P. W. M. Blom, Phys. Rev. B 60, R8489 (1999).

${ }^{7}$ S. Berleb and W. Brütting, Phys. Rev. Lett. 89, 286601 (2002).

${ }^{8}$ S. L. M. van Mensfoort and R. Coehoorn, Phys. Rev. Lett. 100, 086802 (2008).

${ }^{9}$ E. Ehrenfreund, C. Lungenschmied, G. Dennler, H. Neugebauer, and N. S. Sariciftci, Appl. Phys. Lett. 91, 012112 (2007).

${ }^{10}$ S. Berleb, W. Brütting, and G. Paasch, Org. Electron. 1, 41 (2000).

${ }^{11}$ Product datasheet available at http://www.clevios.com.

${ }^{12}$ D. M. Taylor and H. L. Gomes, J. Phys. D 28, 2554 (1995).

${ }^{13}$ S. Nowy, W. Ren, J. Wagner, J. A. Weber, and W. Brütting, Proc. SPIE
7415, 74150G (2009).

${ }^{14}$ W. Brütting, S. Berleb, and A. G. Mückl, Org. Electron. 2, 1 (2001).

${ }^{15}$ W. Brütting, H. Riel, T. Beierlein, and W. Riess, J. Appl. Phys. 89, 1704 (2001).

${ }^{16}$ D. Y. Kondakov, J. R. Sandifer, C. W. Tang, and R. H. Young, J. Appl. Phys. 93, 1108 (2003).

${ }^{17}$ Y. Noguchi, N. Sato, Y. Tanaka, Y. Nakayama, and H. Ishii, Appl. Phys. Lett. 92, 203306 (2008).

${ }^{18}$ Y. Luo, H. Aziz, G. Xu, and Z. D. Popovic, J. Appl. Phys. 101, 054512 (2007).

${ }^{19}$ N. Koch (private communication), 2009.

${ }^{20}$ N. Koch and A. Vollmer, Appl. Phys. Lett. 89, 162107 (2006).

${ }^{21}$ S. T. Lee, Z. Q. Gao, and L. S. Hung, Appl. Phys. Lett. 75, 1404 (1999).

${ }^{22}$ T. Yamada, D. Zou, H. Jeong, Y. Akaki, and T. Tsutsui, Synth. Met. 111112, 237 (2000).

${ }^{23}$ M. Yahiro, D. Zou, and T. Tsutsui, Synth. Met. 111-112, 245 (2000).

${ }^{24}$ J. C. Scott, J. H. Kaufman, P. J. Brock, R. DiPetro, J. Salem, and J. A. Goitia, J. Appl. Phys. 79, 2745 (1996).

${ }^{25}$ F. Papadimitrakopoulos, X.-M. Zhang, D. L. Thomsen, and K. A. Higginson, Chem. Mater. 8, 1363 (1996).

${ }^{26}$ H. Aziz, Z. D. Popovic, N.-X. Hu, A.-M. Hor, and G. Xu, Science 283, 1900 (1999).

${ }^{27}$ Z. D. Popovic, H. Aziz, N.-X. Hu, A. Ioannidis, and P. N. M. dos Anjos, J. Appl. Phys. 89, 4673 (2001).

${ }^{28}$ D. Y. Kondakov, W. C. Lenhart, and W. F. Nichols, J. Appl. Phys. 101, 024512 (2007)

${ }^{29}$ S. Egusa, A. Miura, N. Gemma, and M. Azuma, Jpn. J. Appl. Phys., Part 1 33, 2741 (1994).

${ }^{30}$ D. Y. Kondakov, J. Appl. Phys. 104, 084520 (2008).

${ }^{31}$ V. V. Jarikov and D. Y. Kondakov, J. Appl. Phys. 105, 034905 (2009)

${ }^{32}$ Strictly speaking, one has to distinguish between the detected onset voltage $V_{\text {on }}$ of the current or the luminance, the peak voltage $V_{\text {peak }}$ observed in $C$ - $V$ measurements and the built-in voltage $V_{\mathrm{bi}}$ given by the difference in work function of the anode and cathode material. As pointed out in a recent publication by van Mensfoort et al. (Ref. 8) due to the contribution of diffusion currents and disorder the experimental quantities $V_{\text {on }}$ and $V_{\text {peak }}$ can be lower than $V_{\text {bi }}$ by some tenths of a volt. However, for the sake of simplicity we will not make this distinction here. 